\title{
Temperature-dependent micellar structures in poly(styrene-b-isoprene) diblock copolymer solutions near the critical micelle temperature
}

\author{
Joona Bang, Karthik Viswanathan, and Timothy P. Lodge ${ }^{\text {a) }}$ \\ Department of Chemical Engineering \& Materials Science, University of Minnesota, Minneapolis, \\ Minnesota 55455-0431 and Department of Chemistry, University of Minnesota, Minneapolis, \\ Minnesota 55455-0431 \\ Moon Jeong Park and Kookheon Char \\ School of Chemical Engineering, Seoul National University, Seoul 151-744, Republic of Korea
}

(Received 23 June 2004; accepted 13 September 2004)

\begin{abstract}
The temperature dependence of the micelle structures formed by poly(styrene- $b$-isoprene) (SI) diblock copolymers in the selective solvents diethyl phthalate (DEP) and tetradecane (C14), which are selective for the PS and PI blocks, respectively, have been investigated by small angle neutron scattering (SANS). Two nearly symmetric SI diblock copolymers, one with a perdeuterated PS block and the other with a perdeuterated PI block, were examined in both DEP and C14. The SANS scattering length density of the solvent was matched closely to either the core or the corona block. The resulting core and corona contrast data were fitted with a detailed model developed by Pedersen and co-workers. The fits provide quantitative information on micellar characteristics such as aggregation number, core size, overall size, solvent fraction in the core, and corona thickness. As temperature increases, the solvent selectivity decreases, leading to substantial solvent swelling of the core and a decrease in the aggregation number and core size. Both core and corona chains are able to relax their conformations near the critical micelle temperature due to a decrease in the interfacial tension, even though the corona chains are always under good solvent conditions. (C) 2004 American Institute of Physics. [DOI: 10.1063/1.1812753]
\end{abstract}

\section{INTRODUCTION}

Block copolymer micelles have received much attention in recent years. ${ }^{1,2}$ One appealing advantage of these systems is their versatility; structural details may be tuned by changing the block composition, chain architecture, solvent selectivity, or temperature. They are typically described as a dense core of insoluble blocks and a diffuse corona of soluble blocks, although solvation of the core is also possible depending on the solvent selectivity. ${ }^{2,3}$ One key effect of solvent selectivity (or equivalently, temperature) is that the micelles dissolve into single chains at a critical micelle temperature (CMT) as the solvent selectivity decreases. Several studies have investigated the detailed structure of micelles with varying temperature in aqueous solutions. ${ }^{4-17}$

Small angle neutron (SANS) and x-ray (SAXS) scattering are powerful techniques to characterize micellar structures. ${ }^{18}$ SANS has the particular advantage that the contrast can be readily adjusted by deuteration of the desired block and/or solvent. However, in order to quantify the micellar structure, a detailed fitting model is required. A number of studies have attempted to develop form factors for block copolymer micelles, and particularly the radial density profile of the corona. The corona contributions have been described in several different ways. Examples include a uniform corona shell, ${ }^{6-10}$ a power-law density profile, ${ }^{19,20}$ a Gaussian profile in the "cap and gown" model, ${ }^{11,12}$ and a

\footnotetext{
a) Author to whom correspondence should be addressed. Electronic mail: lodge@chem.umn.edu
}

Fermi-Dirac type density profile. ${ }^{21}$ Recently, a great deal of progress has been achieved by Pedersen and co-workers in obtaining analytical expressions for the form factor based on Monte Carlo simulations. ${ }^{15,22-27}$ This model has been successfully applied to various systems regardless of micellar characteristics (i.e., crew-cut or hairy micelles) and chemical nature (i.e., aqueous or organic systems). ${ }^{13-17,24,28}$

Upon increasing concentration, spherical micelles pack onto a cubic lattice. The resulting structure has been shown to be either an fcc or bcc lattice, depending on the intermicellar potential. ${ }^{29-32} \mathrm{We}$ have recently reported the thermoreversible transition from fcc to bcc upon heating in several poly(styrene- $b$-isoprene) (SI) diblock copolymer solutions, in both S and I selective solvents. ${ }^{33-35}$ By use of in situ shear SAXS, this transition was found to be epitaxial, and the transformation mechanism has been described in detail. ${ }^{34,35}$ One interesting feature is that the transformation pathway is identical to that established in atomic systems. To investigate the cause of this transition, i.e., why bcc becomes favored over fcc upon heating, we characterized the micellar structure with increasing temperature by SANS ${ }^{28}$ A core contrast system was chosen to focus on the core characteristics such as the aggregation number, the core radius, and the solvent fraction in the core. By comparing with recent simulations of highly branched star polymers, the transition was found to be driven by the decreasing aggregation number as the solvent selectivity decreases with increasing temperature. A decrease in the aggregation number leads to a softer intermicellar potential, favoring the bcc lattice. 
In this work we extend this approach to corona contrast systems, in order to characterize the corona density profile more fully. With a combination of the information from core and corona contrast systems, a clear picture on how the solvent selectivity controls the detailed micellar structure can be obtained.

\section{EXPERIMENT}

\section{A. Materials}

Nearly symmetric SI diblock copolymers were synthesized by sequential living anionic polymerization using standard procedures: ${ }^{33}$ one with protonated styrene and perdeuterated isoprene, designated $\operatorname{SdI}(15-14)$, and the other with perdeuterated styrene and protonated isoprene, designated dSI(16-15). The deuterated monomers were purchased from Polymer Source, Inc. Styrene (protonated or perdeuterated) was purified by stirring over calcium hydride for $12 \mathrm{~h}$, followed by vacuum distillation with $n$-butyl lithium for $6 \mathrm{~h}$. Isoprene (protonated or perdeuterated) was treated with dibutyl magnesium for $3 \mathrm{~h}$, followed by $n$-butyl lithium for $6 \mathrm{~h}$. Cyclohexane was used as the polymerization solvent and was distilled from $n$-butyl lithium. Using sec-butyl lithium as an initiator, the styrene was polymerized for $4 \mathrm{~h}$ at $45^{\circ} \mathrm{C}$, followed by the addition of isoprene and the polymerization for $4 \mathrm{~h}$ at the same temperature.

The polymers were characterized by size exclusion chromatography (SEC), using both refractive index and multiangle light scattering detectors (Wyatt Optilab and Dawn), and by ${ }^{1} \mathrm{H}$ nuclear magnetic resonance (NMR). SEC gave the number average block molecular weights, and polydispersities of 1.04 for both $\operatorname{SdI}(15-14)$ and dSI(16-15). The resulting block molecular weights were 15400 (S) and 14100 (dI) $\mathrm{g} / \mathrm{mol}$ for $\operatorname{SdI}(15-14)$, respectively, and $15800(\mathrm{dS})$ and 15400 (I) g/mol for dSI(16-15), respectively. ${ }^{1} \mathrm{H}$ NMR was used to determine the composition and to estimate the mole percent of 4,1-addition of the PI block (94 $1 \%$ ). In addition, a fully protonated SI(15-15), with block molecular weights of 15200 (S) and 15400 (I) g/mol, was used to construct the phase diagrams in Fig. 1.

The solvents diethyl phthalate ( $h$-DEP) and $n$-tetradecane $(h-\mathrm{C} 14)$ were purchased from Aldrich. The deuterated tetradecane $(d$-C14) was obtained from $\mathrm{C} / \mathrm{D} / \mathrm{N}$ Isotope Inc., and mixed with $h$-C14 to match the SANS scattering length density to the desired block. The polymer solutions were prepared gravimetrically, with the aid of methylene chloride as a cosolvent. The cosolvent was stripped off under a stream of nitrogen at room temperature until a constant weight was achieved. The polymer volume fraction $\phi$ was calculated assuming additivity of volumes and densities of $1.118,0.763,0.879,1.047,1.128,0.913$, and $1.021 \mathrm{~g} / \mathrm{cm}^{3}$ for $h$-DEP, $h$-C14, $d$-C14, PS, $d$-PS, PI, and $d$-PI, respectively.

\section{B. Small angle neutron scattering}

Neutron scattering experiments were performed at NIST, Gaithersburg, MD, using the NIST/Exxon/University of Minnesota $30 \mathrm{~m}$ SANS instrument (NG7). Neutrons with a monochromated wavelength $\lambda$ of $6 \AA$ and a wavelength

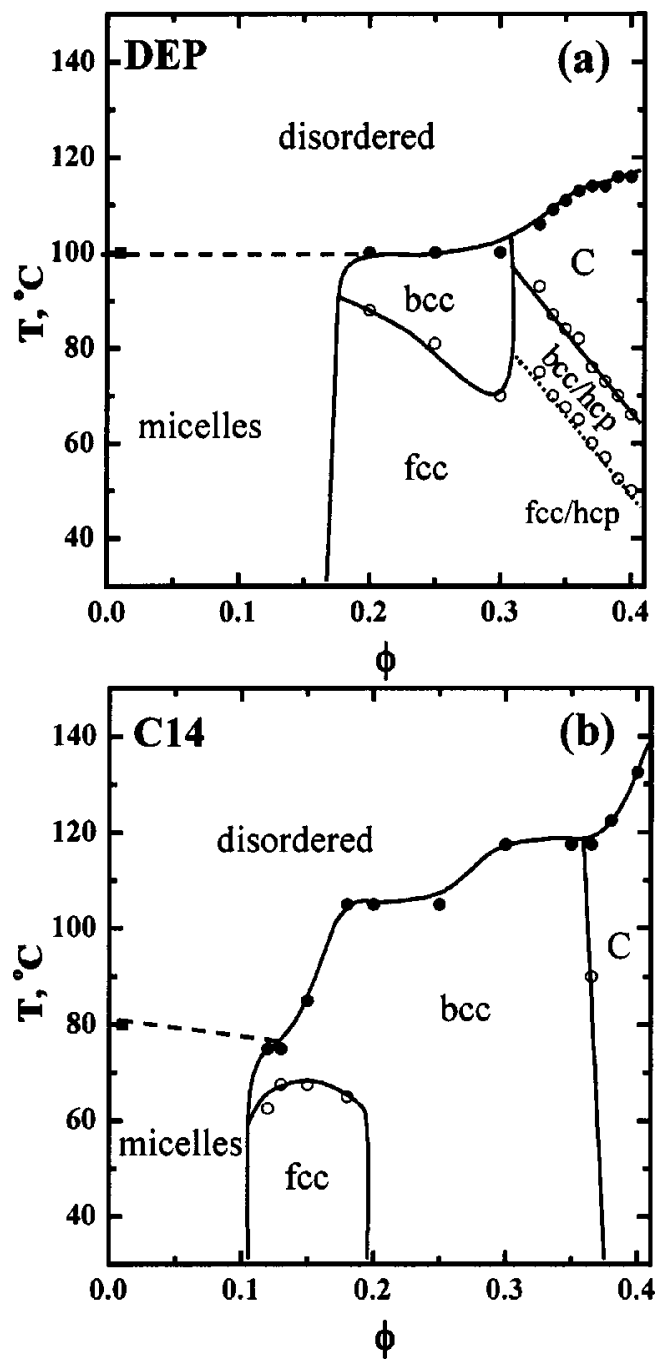

FIG. 1. Phase diagrams for SI(15-15) in (a) DEP and (b) C14 as functions of temperature $T$ and polymer volume fraction $\phi$.

spread $(\Delta \lambda / \lambda)$ of 0.11 were incident on the sample. A sample to detector distance of $7.0 \mathrm{~m}$ was used to access scattering wave vectors $q$ in the range $0.007 \AA^{-1}<q<0.098 \AA^{-1}$. The scattering vector $q$ is defined as $q=4 \pi / \lambda \sin (\theta / 2)$, where $\theta$ is the scattering angle. The isotropic, two-dimensional data were azimuthally averaged to obtain the intensity versus $q$. The resulting data were corrected for detector sensitivity, sample transmission, empty cell scattering, and sample thickness. The scattering intensities were then scaled to absolute values based on the direct beam flux method. Finally, the coherent scattering intensity was obtained after appropriate subtraction of the solvent scattering and of the incoherent background.

Solutions with polymer volume fractions $\phi=0.005,0.01$, and 0.02 were put in sealed quartz "banjo" cells with ca. $1 \mathrm{~mm}$ path length, and examined at $10^{\circ} \mathrm{C}$ intervals upon heating. At each temperature, the solutions were annealed for at least $600 \mathrm{~s}$, and data were collected for 300 or $600 \mathrm{~s}$ depending on the concentration and the contrast. On the basis of the densities of solvents and polymers, the neutron scattering length densities were estimated as $\rho_{\mathrm{PS}}=1.41 \times 10^{10} \mathrm{~cm}^{-2}, \quad \rho_{d-\mathrm{PS}}=6.45 \times 10^{10} \mathrm{~cm}^{-2}, \quad \rho_{\mathrm{PI}}$ 
$=0.27 \times 10^{10} \mathrm{~cm}^{-2}, \quad \rho_{d-\mathrm{PI}}=6.85 \times 10^{10} \mathrm{~cm}^{-2}, \rho_{h \text {-DEP }}=1.53$ $\times 10^{10} \mathrm{~cm}^{-2}, \rho_{h-\mathrm{C} 14}=-0.44 \times 10^{10} \mathrm{~cm}^{-2}$, and $\rho_{d-\mathrm{C} 14}=6.79$ $\times 10^{10} \mathrm{~cm}^{-2}$. Since $\rho_{\text {PS }}$ and $\rho_{\text {PI }}$ are greater than $\rho_{h-\mathrm{C} 14}$ and less than $\rho_{d-\mathrm{C} 14}$, the contrast can be matched to either the PS or PI block by mixing $h$-C14 and $d$-C14. The scattering density for the mixed solvent $\left(\rho_{\mathrm{sol}}\right)$ is $\rho_{\mathrm{sol}}=\phi_{h-\mathrm{C} 14} \rho_{h-\mathrm{C} 14}$ $+\left(1-\phi_{h-\mathrm{C} 14}\right) \rho_{d-\mathrm{C} 14}$, where $\phi_{h-\mathrm{C} 14}$ is the volume fraction of $h$-C14. Thus the contrast can be exactly matched to the core or corona block for $\mathrm{SdI}(15-14)$ in $\mathrm{C} 14$ and $\mathrm{dSI}(16-15)$ in C14, respectively. Unfortunately, $\rho_{\mathrm{PS}}$ and $\rho_{\mathrm{PI}}$ are both less than $\rho_{h \text {-DEP }}$, and deuteration of DEP ( $d$-DEP) will increase the scattering length density further, which does not make it possible to match the solvent contrast to the PS or PI block by mixing $h$-DEP and $d$-DEP. Fortunately, approximate core contrast can be obtained for $\operatorname{SdI}(15-14)$ in DEP solutions as $\rho_{h \text {-DEP }}$ is very close to $\rho_{\mathrm{PS}}$. For dSI(16-15) in DEP, $\rho_{h \text {-DEP }}$ is much closer to $\rho_{\mathrm{PI}}$ than to $\rho_{d \text {-PS }}$, and thus the features from the corona scattering are emphasized.

\section{Dynamic light scattering}

Dynamic light scattering (DLS) was used to determine the CMT and measure the hydrodynamic radius $R_{h}$ of micelles for the same $\phi=0.01$ solutions which were investigated with SANS. Each sample was passed through an 0.2 $\mu \mathrm{m}$ filter (Millipore) into an 0.25 in. diameter optical glass tube. The tube was flame-sealed under vacuum to prevent oxidative degradation and dust contamination. Measurements were taken on a home-built photometer equipped with an electrically heated silicon oil index-matching bath, a Brookhaven BI-DS photomultiplier, a Lexel Ar+ laser operating at $488 \mathrm{~nm}$, and a Brookhaven BI-9000 correlator. Samples were annealed at the set temperature for at least 10 min before intensity autocorrelation functions $g^{(2)}(t)$ were recorded. At each selected temperature, measurements were made at a minimum of three scattering angles from $50^{\circ}$ to $130^{\circ}$. Intensity correlation functions were then fitted to either single exponential decays (micelles or single chains) or a sum of two exponentials ("anomalous micellization" regime), as described elsewhere. ${ }^{36}$

\section{FITTING MODEL}

To fit the SANS data, the scattering form factor for a block copolymer micelle with a spherical core and corona chains attached to the core surface developed by Pedersen and co-workers was applied. ${ }^{15,22,25}$ This model contains four different terms: the self-correlation of the core, the selfcorrelation of the corona chains, the cross term between the core and corona chains, and the cross term between different corona chains. It can be expressed as

$$
\begin{aligned}
P_{\text {mic }}(q)= & Q^{2} \beta_{\text {core }}^{2} A_{\text {core }}^{2}(q)+Q(Q-1) \beta_{\text {corona }}^{2} A_{\text {corona }}^{2}(q) \\
& +2 Q^{2} \beta_{\text {core }} \beta_{\text {corona }} A_{\text {core }}(q) A_{\text {corona }}(q) \\
& +Q \beta_{\text {corona }}^{2} P_{\text {chain }}(q),
\end{aligned}
$$

where $q$ is the scattering vector, $Q$ is the aggregation number, and $\beta_{\text {core }}$ and $\beta_{\text {corona }}$ are the total excess scattering lengths of the core and corona blocks, respectively. They are defined as $\beta_{\text {core }}=v_{\text {core-block }}\left(\rho_{\text {core-block }}-\rho_{\text {sol }}\right)$ and $\beta_{\text {corona }}$ $=v_{\text {corona-block }}\left(\rho_{\text {corona-block }}-\rho_{\text {sol }}\right)$, where $v_{x}$ is the volume of the core or corona block, and $\rho_{\text {core-block }}, \rho_{\text {corona-block }}$, and $\rho_{\text {sol }}$ are the scattering length density of the core block, the corona block, and the solvent, respectively. For a homogeneous spherical core with radius $R_{c}$ and a smoothly decaying scattering length density at the core surface, the core self-term is

$$
A_{\text {core }}^{2}(q)=\Phi^{2}\left(q R_{c}\right) \exp \left(-q^{2} \sigma^{2}\right)
$$

where $\Phi(x)=3[\sin x-x \cos x] / x^{3}$ is the hard-sphere form factor. The exponential term reflects a smoothly decaying density at the core surface, and $\sigma$ is related to the width of the interface.

The corona chain self-correlation term for Gaussian chains with radius of gyration $R_{g}$ is given by the well-known Debye function

$$
P_{\text {chain }}(q)=\frac{2[\exp (-x)-1+x]}{x^{2}},
$$

where $x=q^{2} R_{g}^{2}$. For a micelle modelled with noninteracting Gaussian chains in the corona, the corona term can be written as

$$
\begin{aligned}
A_{\text {corona }}(q)= & \psi\left(q^{2} R_{g}^{2}\right)\left(\frac{\sin \left[q\left(R_{c}+d R_{g}\right)\right]}{q\left(R_{c}+d R_{g}\right)}\right) \\
& \times \exp \left(-q^{2} \sigma^{2} / 2\right),
\end{aligned}
$$

where $\psi(x)=[1-\exp (-x)] / x^{2}$; with $x=q^{2} R_{g}^{2}$. In this equation $d$ is close to unity ( $d \approx 1)$, as this mimics nonpenetration of the corona chains into the core region. In other words, the end of the corona chain can be considered to lie on a surface at $R_{c}+d R_{g}$, not $R_{c}$. While this model has a simple form, the corresponding radial profile is not well defined, as there can be overlap between core and corona. Hence this model was only applied to $\mathrm{SdI}(15-14)$ in DEP solutions where the corona contribution is not significant $\left(\beta_{\text {corona }}^{2} / \beta_{\text {core }}^{2} \approx 0.005\right)$.

For the corona contrast systems, i.e., $\mathrm{SdI}(15-14)$ in $\mathrm{C} 14$ and $\mathrm{dSI}(16-15)$ in DEP, the Gaussian noninteracting model cannot describe the corona density profile properly and one needs a better defined expression. $A_{\text {corona }}(q)$ is then given as the normalized Fourier transform of the radial density distribution function of the corona chains, $\rho_{\text {corona }}(r)$, as follows:

$$
A_{\text {corona }}(q)=\frac{4 \pi \int \rho_{\text {corona }}(r) \frac{\sin (q r)}{q r} r^{2} d r}{4 \pi \int \rho_{\text {corona }}(r) r^{2} d r} \exp \left(-q^{2} \sigma^{2} / 2\right)
$$

In this work, we have chosen to represent $\rho_{\text {corona }}(r)$ as a linear combination of two cubic $b$ splines, as has been successfully applied previously, ${ }^{13-15}$ and the number of fitting parameters increases by only one compared to the noninteracting Gaussian chain model. The explicit form of $\rho_{\text {corona }}(r)$ is described in the Appendix. Note that this expression was first developed by Pedersen et al. ${ }^{15}$

Assuming a hard-sphere structure factor, the total coherent scattering intensity is ${ }^{25}$

$$
I(q)=P_{\text {mic }}(q)+A_{\text {mic }}(q)^{2}[S(q)-1],
$$


where $S(q)$ is the monodisperse hard-sphere structure factor $^{37}$ and $A_{\text {mic }}(q)$ is the form factor amplitude of the radial scattering length distribution of the micelle. It can be expressed as

$$
A_{\text {mic }}(q)=Q\left[\beta_{\text {core }} A_{\text {core }}(q)+\beta_{\text {corona }} A_{\text {corona }}(q)\right] .
$$

For a polydisperse model, a Gaussian distribution for the core radii can be assumed:

$$
D\left(R_{c}\right)=\frac{1}{\sqrt{2 \pi} \sigma_{R}} \exp \left[\frac{-\left(R_{c}-\left\langle R_{c}\right\rangle\right)^{2}}{2 \sigma_{R}^{2}}\right] \text { for } R_{c}>0,
$$

where $\left\langle R_{c}\right\rangle$ is the average radius and $\sigma_{R}$ is the width of the distribution. The coherent scattering intensity for the polydisperse model is then

$$
I(q)=\int D\left(R_{c}\right)\left(P_{\mathrm{mic}}(q)+A_{\mathrm{mic}}(q)^{2}[S(q)-1]\right) d R_{c} .
$$

This expression is known as "local monodisperse approximation" and was derived by Pedersen. ${ }^{38}$ The structure factor $S(q)$ depends on two parameters, the hard-sphere radius $R_{h s}$ and the hard-sphere volume fraction $\phi_{h s} .{ }^{37}$

Another factor to be considered in fitting the SANS data is the smearing which results from instrumental limitations. The smeared intensity $I_{s}(\bar{q})$ can be calculated via a convolution of $I(q)$ with an instrumental resolution function $R(q, \bar{q})$ as

$$
I_{s}(\bar{q})=\int R(q, \bar{q}) I(q) d q .
$$

The resolution function is approximated by a Gaussian distribution of $q$ at an average value $\bar{q}: 39,40$

$$
R(q, \bar{q}) \equiv \frac{f_{s}}{\sqrt{2 \pi V_{q}}} \exp \left[\frac{-(q-\bar{q})^{2}}{2 V_{q}}\right],
$$

where $f_{s}$ is the shadow factor which accounts for beam-stop shadowing effects, ${ }^{41} \bar{q}$ is the mean scattering vector, and $V_{q}\left(=\sigma_{q}^{2}\right)$ is the variance. These are determined from the wavelength distribution, apertures, detector resolution, etc. 39,40

For the core contrast systems, the fitting parameters are the aggregation number $Q$, the hard-sphere volume fraction $\phi_{h s}$, the hard-sphere radius $R_{h s}$, the core radius $R_{c}$, and the width of the distribution $\sigma_{R}$. The number of fitting parameters for the corona contrast systems increases by four: the radius of gyration of the corona chains $R_{g}$, the width of the core-corona interface $\sigma$, and $a_{1}$ and $s$ in the corona term (see the Appendix).

\section{RESULTS}

\section{A. Phase diagram}

We have previously mapped out the phase diagrams of several SI diblock copolymers in both S and I selective solvents as a function of polymer volume fraction and temperature. ${ }^{33,42-44}$ By a combination of SAXS, rheology, and static birefringence, various temperature-dependent order-order transitions (OOT) have been located and identi- fied. Thermotropic OOTs can be understood in terms of changes in the solvent selectivity, namely, a decrease in solvent selectivity with increasing temperature gives rise to different partitioning of solvent between the two microdomains, resulting in changes in both interfacial tension and interfacial curvature. We have recently focused on the thermoreversible fcc to bec transition, and the fcc/bcc phase boundary was located in several SI solutions. ${ }^{34,35}$

For a symmetric SI copolymer, the thermotropic as well as lyotropic phase behavior in both $\mathrm{S}$ and I selective solvents are expected to be similar. Figure 1 shows the phase diagrams for SI(15-15) in DEP and C14 over the polymer volume fraction range $\phi=0-0.4$. The observed phases are disordered micelles, cubic lattices of spherical micelles (fcc or bcc), and cylinders (C) with increasing $\phi$. Even though the fcc to bcc transition appears over a range of $\phi$ in both solutions, the detailed phase behavior of the cubic lattices are quite different. For DEP solutions, the fcc to bcc transition persists up to $\phi \approx 0.3$, then a closed-packed sphere (fcc and hcp) to cylinder transition was observed at higher $\phi$, whereas in $\mathrm{C} 14$ the fcc to bcc transition appears at lower $\phi$ and a broad window of bcc exists, followed by the bcc to cylinder transition for $\phi \geqslant 0.36$. Note that the closed-packed sphere to cylinder transition shown in DEP solutions was found to follow quite complicated pathways, and the associated epitaxial relationships are reported elsewhere. ${ }^{45}$ The differences in phase behavior between DEP and C14 solutions presumably come from the intermicellar potential. The micelles in C14 solutions have longer corona blocks, due to the larger radius of gyration of PI vs PS at equal molecular weight, which favors bcc rather than fcc. ${ }^{29-32}$ This difference will be quantified later by comparing the ratio of the corona layer thickness to the core radius, $L_{\text {corona }} / R_{c}$.

In dilute solutions, i.e., $\phi \approx 0.01$, where the intermicellar interaction is almost negligible, the CMT and $R_{h}(\mathrm{~T})$ were characterized by DLS. One notable feature is that the low $\phi$ CMT is very close to the higher $\phi$ ODT, where the bcc to disorder transition occurs, as shown in Fig. 1. This phenomenon was consistently observed in all the phase diagrams we have constructed, ${ }^{33,35,42}$ which suggests that the temperaturedependent micellar behavior at low $\phi$ and in the ordered state at higher $\phi$ track each other closely. ${ }^{28}$ Information on how the change in the solvent selectivity with increasing temperature affects the micellar structure can be obtained in dilute solutions, and this can be directly applied to the higher $\phi$ solutions to understand the cause of the fcc to bcc transition. Since DLS only measures the temperature-dependent $R_{h}$, more detailed micellar characteristics (such as aggregation number, core radius, corona thickness, and solvent fraction in the core) were obtained by SANS, as will be described in the following sections. In this case, $\operatorname{SdI}(15-14)$ or dSI(16-15) were employed to enhance the SANS contrast. Since the molecular weights and block compositions of SdI(15-14) and dSI(16-15) are very close to those of SI(15$15)$, we can assume that the phase behavior of $\operatorname{SdI}(15-14)$ and dSI(16-15) in DEP or C14 would be very similar to those shown in Fig. 1. 


\section{B. Core contrast condition}

Four different systems have been investigated: $\operatorname{SdI}(15-$ 14) in DEP and dSI(16-15) in C14 (core contrast), and dSI(16-15) in DEP and SdI(15-14) in C14 (corona contrast) solutions. Figure 2 displays the corresponding form factors measured at $30^{\circ} \mathrm{C}$ for three systems, for three different concentrations $(\phi=0.005,0.01$, and 0.02$)$. The corresponding data for the fourth system, dSI(16-15) in C14, were presented previously. ${ }^{28}$ As the concentration increases, the first minimum and maximum in the form factor become more distinct in all cases. In Fig. 2(a), the peak from the structure factor also emerges near $q \approx 0.01 \AA^{-1}$ at $\phi=0.02$, as the intermicellar interaction increases. Figure 3 shows the temperature dependence of the form factors for the three systems with $\phi=0.01$. The corresponding data for $\operatorname{SdI}(15-14)$ in DEP was presented previously. ${ }^{28}$ The main features are that the first minimum and the secondary maximum are smeared out and the intensity at low $q$ decreases, with increasing temperature. The form factors for $\mathrm{dSI}(16-15)$ in $\mathrm{C} 14$ with $\phi=0.005$, 0.01 , and 0.02 and $\operatorname{SdI}(15-14)$ in DEP with increasing temperature show the same features as Figs. 2(a) and 3(a), respectively. All of the smooth curves in Figs. 2 and 3 represent the best fits to the expressions given in the preceding section, as will be discussed subsequently. All of the fitting parameters are listed in Tables I (core contrast) and II (corona contrast).

For the core contrast systems, Fig. 2(a) is a representative example of the hard-sphere form factor, with a distinct first minimum at $0.045 \AA^{-1}$. From the characteristic equation for minima in the hard-sphere form factor, $\sin \left(q R_{c}\right)$ $-q R_{c} \cos \left(q R_{c}\right)=0$, the core radius can be estimated as $100 \AA$ with $q R_{c}=4.493$ at the first minimum. In this case, the fits were facilitated as the corona contribution is negligible. Figure 3(a) shows the scattering form factors and the corresponding fits for dSI(16-15) in C14 $\phi=0.01$ with increasing temperature. The scattering data were modeled by taking only the first term in Eq. (1) for $\mathrm{dSI}(16-15)$ in $\mathrm{C} 14$, as it satisfies the core-contrast condition exactly $\left(\beta_{\text {corona }}=0\right)$. For SdI(15-14) in DEP, we also considered the corona terms, as the corona blocks were not exactly masked. The fitting parameters are the aggregation number $Q$, the effective hardsphere volume fraction $\phi_{h s}$, the effective hard-sphere radius $R_{h s}$, the core radius $R_{c}$, and the core size distribution $\sigma_{R}$. For SdI(15-14) in DEP, one needs to also consider the radius of gyration of the corona chains $\left(R_{g}\right)$ and the displacement of the corona chains $(d)$, but it was assumed that $R_{g}$ is equal to $L_{\text {corona }} / 2$ and $d=1$, as the fit is essentially insensitive to those parameters $\left(\beta_{\text {corona }}^{2} / \beta_{\text {core }}^{2} \approx 0.005\right)$. Hence the number of the fitting parameters is same in both systems. $R_{h s}$ and $\phi_{h s}$ can be obtained from the hard-sphere structure factor, and the corona layer thickness was calculated via $L_{\text {corona }}$ $=R_{h s}-R_{c}$. The resulting fit parameters are summarized in Table I.

The aggregation number $Q$ can be directly determined from the scattering intensity at low $q$, and a decrease in the intensity at low $q$ with increasing temperature results in the decrease in $Q$ [Figs. 4(a) and 4(b)]. In both solutions, $Q$ decreases from ca. 180 at $30^{\circ} \mathrm{C}$ to ca. 60 near the CMT. Also
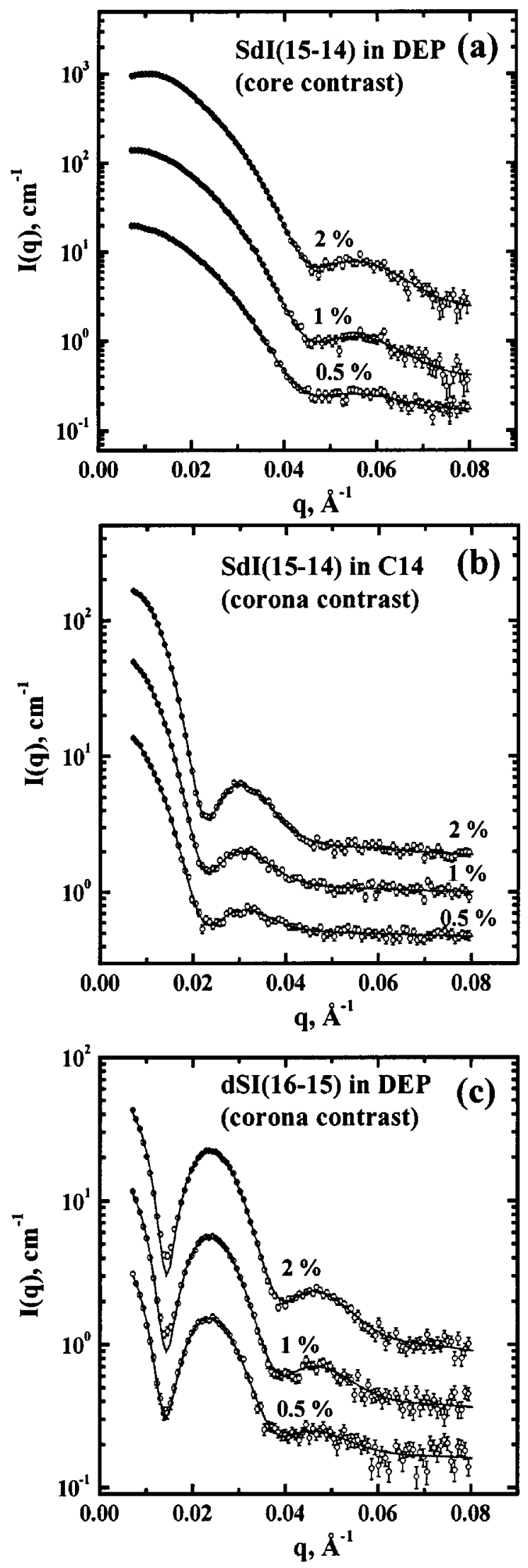

FIG. 2. SANS data as a function of concentration for SdI(15-14) in (a) DEP and (b) C14, and (c) dSI(16-15) in DEP at $30^{\circ} \mathrm{C}$. For clarity $\phi=0.01$ and 0.02 data have been multiplied by 4 and 16 for (a) or by 2 and 4 for (b) and (c), respectively. The symbols are the SANS data, and the solid lines are the model fits. 

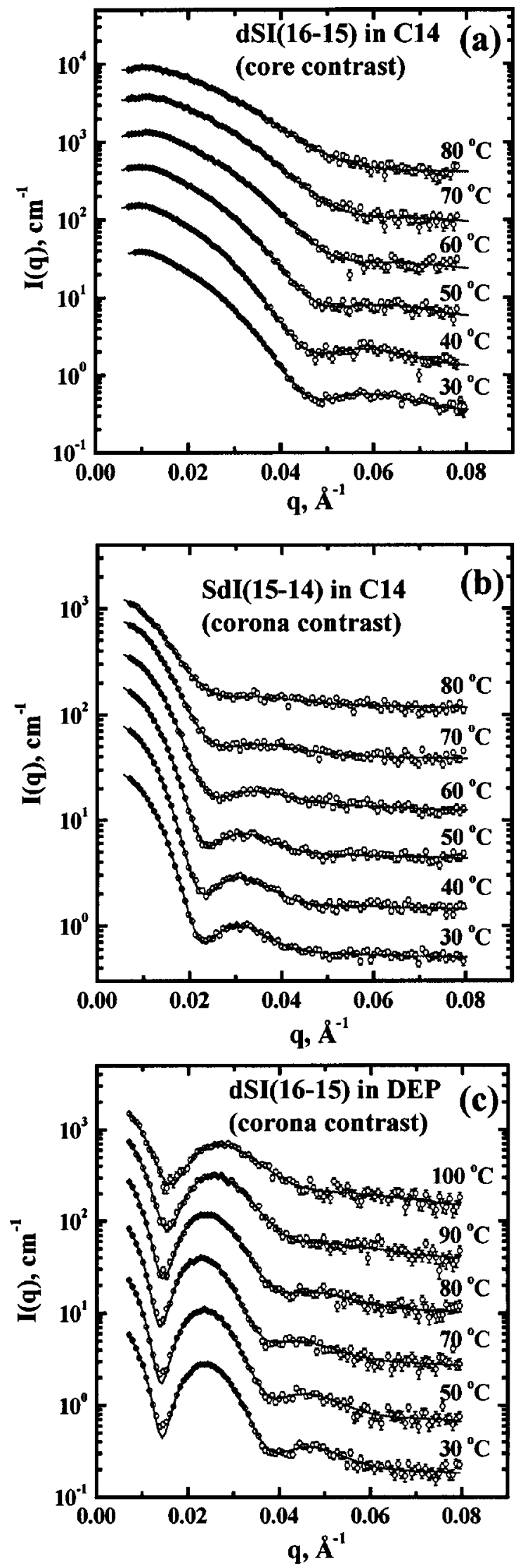

FIG. 3. SANS data as a function of temperature for (a) dSI(16-15) in C14 $\phi=0.01$, (b) $\mathrm{SdI}(15-14)$ in $\mathrm{C} 14 \phi=0.01$, and (c) $\mathrm{dSI}(16-15)$ in DEP $\phi=0.01$. For clarity the data have been multiplied by $4,4^{2}, 4^{3}, 4^{4}$, and $4^{5}$, respectively. The symbols are the SANS data, and the solid lines are the model fits. note that $Q$ is not affected by the concentration, as noted previously. ${ }^{28}$ The key factor controlling the micellar structure in this system is the temperature-induced change in the solvent selectivity. As temperature increases, the solvent becomes less selective, i.e., the effective interaction parameter between the core block and the solvent, $\chi_{\text {core-sol }}$, becomes smaller, and therefore the solvent can penetrate the core. The solvent fraction in the core, $\phi_{\text {core,sol }}$, was calculated using the fitted values via $4 / 3 \pi R_{c}^{3}=Q v_{\text {core-block }} /\left(1-\phi_{\text {core,sol }}\right)$. The fits indicate that there is essentially no solvent in the core for both solutions at low temperature, and that the solvent begins to penetrate the core increasingly with increasing temperature. In the proximity of the CMT, the micelles are swollen up to $\phi_{\text {core,sol }} \approx 0.3$, as shown in Figs. 4(c) and 4(d).

At elevated temperature one expects the micellar coronas to become less crowded, due to the decrease in $Q$. Also, the interfacial tension at the core-corona interface will be reduced due to the solvent in the core, which allows both core and corona chains to relax. As a result, one expects that $R_{c}$ and $L_{\text {corona }}$ (or $R_{g}$ ) will decrease with increasing temperature [Figs. 5(a-d)], consistent with the fit results. As with $Q$, the $R_{c}$ values are also nearly independent of concentration. The fits show that $L_{\text {corona }}$ also decreases, but the data show some scatter depending on the concentration. This is probably because $L_{\text {corona }}$ is determined from the hard-sphere structure factor, and there could be significant uncertainty due to the weak correlations among distinct spheres in these dilute solutions. More reliable information on the corona chains can be obtained from the corona contrast systems. However, $L_{\text {corona }}$ values do exhibit the expected trend in that the corona chains relax due to a decrease in the interfacial tension with increasing temperature. The ratio of the corona layer thickness to the core radius, $L_{\text {corona }} / R_{c}$, has been used to classify the intermicellar potential. ${ }^{29,30,46}$ In our system, $L_{\text {corona }} / R_{c}$ is essentially constant over the entire temperature range [Figs. 5(e) and 5(f)], implying that this criterion is not really a good way to quantify the temperature-induced changes in the intermicellar potential. The values are between 0.75 and 0.85 for $\mathrm{SdI}(15-14)$ in DEP, and between 1.2 and 1.4 for dSI(16-15) in C14. Consequently, dSI(16-15) in $\mathrm{C} 14$ has the longer-ranged intermicellar potential than SdI(15-14) in DEP, and this supports the fact that the bcc phase is observed over a wider $\phi$ range in the phase diagram of SI(15-15) in C14 solutions shown in Fig. 1. But, in both cases the fcc to bcc transition is accessed while $L_{\text {corona }} / R_{c}$ is constant.

\section{Corona contrast condition}

The scattering profiles for the corona contrast condition are significantly different from the core contrast condition. In Fig. 2(b) $\mathrm{SdI}(15-14)$ in $\mathrm{C} 14$, the solvent contrast is exactly matched to the core by mixing $h-\mathrm{C} 14$ and $d-\mathrm{C} 14$, and hence the first and third terms in Eq. (1) can be eliminated. In this case, the characteristic equation becomes $\sin \left[q\left(R_{c}+d R_{g}\right)\right]$ $=0$ for the model with noninteracting Gaussian chains. With $q\left(R_{c}+d R_{g}\right)=3.142$ and $q=0.023 \AA^{-1}$ at the first minimum, $R_{c}+d R_{g}$ is $137 \AA$, whereas the fit results give $R_{c}+d R_{g}$ of 
TABLE I. Fit results for core contrast systems.

\begin{tabular}{|c|c|c|c|c|c|c|c|c|c|c|c|c|}
\hline \multirow[b]{2}{*}{$T\left({ }^{\circ} \mathrm{C}\right)$} & \multicolumn{8}{|c|}{$\begin{array}{l}\mathrm{SdI}(15-14) \text { in DEP } \\
\phi=0.01\end{array}$} & \multicolumn{4}{|c|}{$\phi=0.02$} \\
\hline & $Q$ & $R_{c}(\AA)$ & $L_{\text {corona }}(\AA)$ & $\sigma_{R}(\AA)$ & $Q$ & $R_{c}(\AA)$ & $L_{\text {corona }}(\AA)$ & $\sigma_{R}(\AA)$ & $Q$ & $R_{c}(\AA)$ & $L_{\text {corona }}(\AA)$ & $\sigma_{R}(\AA)$ \\
\hline 30 & 173 & 102 & 88 & 16 & 184 & 103 & 85 & 16 & 165 & 102 & 78 & 16 \\
\hline 40 & 165 & 101 & 88 & 17 & 160 & 103 & 78 & 17 & 157 & 103 & 79 & 17 \\
\hline 50 & 115 & 93 & 80 & 17 & 121 & 97 & 73 & 18 & 121 & 97 & 75 & 18 \\
\hline 60 & 88 & 95 & 71 & 23 & 99 & 93 & 70 & 20 & 95 & 92 & 72 & 20 \\
\hline \multirow[t]{2}{*}{70} & $\ldots$ & $\cdots$ & $\cdots$ & $\cdots$ & 67 & 86 & 65 & 29 & 68 & 85 & 67 & 22 \\
\hline & \multicolumn{8}{|c|}{$\begin{array}{r}\mathrm{dSI}(16-15) \text { in } \mathrm{C} 14 \\
\phi=0.01\end{array}$} & \multicolumn{4}{|c|}{$\phi=0.02$} \\
\hline$T\left({ }^{\circ} \mathrm{C}\right)$ & $Q$ & $R_{c}(\AA)$ & $L_{\text {corona }}(\AA)$ & $\overline{\sigma_{R}(\AA)}$ & $Q$ & $R_{c}(\AA)$ & $L_{\text {corona }}(\AA)$ & $\overline{\sigma_{R}(\AA)}$ & $Q$ & $R_{c}(\AA)$ & $L_{\text {corona }}(\AA)$ & $\sigma_{R}(\AA)$ \\
\hline 30 & 157 & 98 & 117 & 15 & 161 & 98 & 132 & 14 & 180 & 101 & 131 & $\overline{15}$ \\
\hline 40 & 155 & 99 & 122 & 16 & 168 & 99 & 131 & 15 & 178 & 102 & 132 & 15 \\
\hline 50 & 136 & 96 & 127 & 16 & 134 & 94 & 122 & 15 & 132 & 94 & 125 & 15 \\
\hline 60 & 90 & 88 & 105 & 17 & 94 & 88 & 110 & 17 & 94 & 87 & 116 & 16 \\
\hline 70 & 81 & 85 & 118 & 18 & 77 & 83 & 103 & 17 & 77 & 83 & 110 & 17 \\
\hline 80 & 58 & 84 & 101 & 22 & 63 & 79 & 107 & 18 & 59 & 80 & 100 & 19 \\
\hline
\end{tabular}

$158 \AA\left(R_{c}=98 \AA, R_{g}=60 \AA\right.$, and $\left.d=1\right)$. The discrepancy may come from the complication of the corona profile, as a set of two cubic $b$ spline functions was incorporated rather than the noninteracting Gaussian chain model. With the two cubic $b$ spline functions, the characteristic equation becomes $A_{\text {corona }}\left(q, R_{c}, s, a_{1}\right)=0$. Inserting fitted values at $\phi=0.01,98$ $\AA$, $76 \AA$, and 0.042 for $R_{c}, s$, and $a_{1}$, respectively, the equation gives $q=0.023 \AA^{-1}$, reflecting that the minimum of the form factor follows the characteristic equation.

The situation becomes more complicated in $\mathrm{dSI}(16-15)$ in DEP [Fig. 2(c)]. The scattering patterns show a pronounced first maximum and a sharp minimum at lower $q$. The contrasts for the core and corona are -1.261 $\times 10^{-10} \mathrm{~cm}^{-2}\left(\rho_{\mathrm{PI}}-\rho_{\mathrm{DEP}}\right)$ and $4.92 \times 10^{-10} \mathrm{~cm}^{-2}\left(\rho_{d-\mathrm{PS}}\right.$
$\left.-\rho_{\mathrm{DEP}}\right)$, respectively, and $\beta_{\text {core }}^{2} / \beta_{\text {corona }}^{2} \approx 0.08$. Hence all the terms in Eq. (1) have a significant contribution to the scattering pattern, and the characteristic equation cannot be simply extracted. Despite this complexity, we suggest that the main features can be ascribed to the contribution from the negative term, i.e., the cross term between the core and corona $\left(\beta_{\text {core }} \beta_{\text {corona }}<0\right)$, as evidenced by simulations shown in Fig. 6. In this case micelles were considered to consist of symmetric diblock copolymers with equal block volumes, $30000 \AA^{3} /$ block, and a core radius $R_{c}$ of $110 \AA$. The aggregation number $Q$ was calculated assuming there is no solvent in the core $(Q=186)$. For the corona part, the Gaussian noninteracting model was considered for simplicity and $R_{g}$ $=45 \AA$ and $d=1$ were taken. The resulting micellar dimen-

TABLE II. Fit results for corona contrast systems.

\begin{tabular}{|c|c|c|c|c|c|c|c|c|c|c|c|c|}
\hline \multirow[b]{3}{*}{$T\left({ }^{\circ} \mathrm{C}\right)$} & \multicolumn{9}{|c|}{$\mathrm{dSI}(16-15)$ in DEP } & & & \\
\hline & & & & & & & \multicolumn{6}{|c|}{$\phi=0.02$} \\
\hline & $Q$ & $R_{c}(\AA)$ & $R_{g}(\AA)$ & $a_{1}$ & $s(\AA)$ & $\sigma_{R}(\AA)$ & $Q$ & $R_{c}(\AA)$ & $R_{g}(\AA)$ & $a_{1}$ & $s(\AA)$ & $\sigma_{R}(\AA)$ \\
\hline 30 & 119 & 114 & 44 & -0.15 & 93 & 12 & 149 & 116 & 42 & -0.13 & 92 & 15 \\
\hline 40 & 127 & 115 & 45 & -0.14 & 93 & 16 & 152 & 117 & 43 & -0.12 & 93 & 15 \\
\hline 50 & 126 & 116 & 45 & -0.13 & 94 & 15 & 153 & 118 & 45 & -0.11 & 94 & 16 \\
\hline 60 & 127 & 119 & 47 & -0.17 & 91 & 17 & 147 & 121 & 42 & -0.06 & 95 & 17 \\
\hline 70 & 120 & 117 & 46 & -0.20 & 91 & 16 & 152 & 123 & 45 & -0.05 & 96 & 18 \\
\hline 80 & 107 & 111 & 41 & -0.09 & 95 & 17 & 126 & 114 & 43 & -0.08 & 93 & 19 \\
\hline 90 & 77 & 101 & 34 & -0.06 & 95 & 17 & 99 & 104 & 39 & -0.19 & 85 & 20 \\
\hline \multirow[t]{3}{*}{100} & 38 & 96 & 21 & 0.23 & 104 & 16 & 64 & 94 & 32 & -0.04 & 94 & 19 \\
\hline & \multirow{2}{*}{\multicolumn{12}{|c|}{$\mathrm{SdI}(15-14)$ in $\mathrm{C} 14$}} \\
\hline & & & & & & & & & & & & \\
\hline$T\left({ }^{\circ} \mathrm{C}\right)$ & $Q$ & $R_{c}(\AA)$ & $R_{g}(\AA)$ & $a_{1}$ & $s(\AA)$ & $\sigma_{R}(\AA)$ & $Q$ & $R_{c}(\AA)$ & $R_{g}(\AA)$ & $a_{1}$ & $s(\AA)$ & $\sigma_{R}(\AA)$ \\
\hline 30 & 155 & 98 & 60 & 0.04 & 76 & 12 & 155 & 98 & 60 & 0.05 & 80 & 12 \\
\hline 40 & 155 & 98 & 64 & 0.20 & 84 & 12 & 154 & 98 & 60 & -0.06 & 76 & 13 \\
\hline 50 & 131 & 94 & 60 & 0.22 & 80 & 12 & 120 & 94 & 56 & -0.20 & 53 & 9 \\
\hline 60 & 89 & 87 & 50 & 0.60 & 92 & 9 & 89 & 87 & 52 & 0.02 & 66 & 8 \\
\hline 70 & 76 & 83 & 48 & 0.56 & 96 & 19 & 73 & 83 & 45 & -0.22 & 52 & 4 \\
\hline 80 & 63 & 79 & 48 & 2.72 & 133 & 32 & 63 & 80 & 50 & 0.23 & 77 & 20 \\
\hline
\end{tabular}




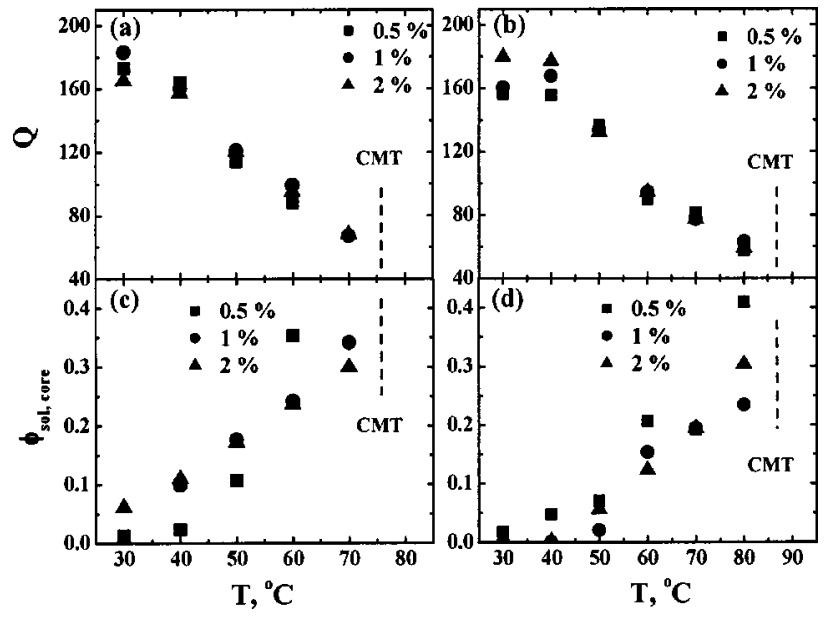

FIG. 4. Temperature dependence of the aggregation number $Q$ for (a) $\operatorname{SdI}(15-14)$ in DEP and (b) dSI(16-15) in C14, and the solvent volume fraction in the core, $\phi_{\text {sol,core }}$, for (c) SdI(15-14) in DEP and (d) dSI(16-15) in C14.

sion is very close to that of $\mathrm{dSI}(16-15)$ in DEP solution. $\rho_{\text {corona-block }}$ and $\rho_{\text {solvent }}$ were fixed as $6.0 \times 10^{10}$ and 4.0 $\times 10^{10} \mathrm{~cm}^{-2}$, respectively, and $\rho_{\text {core-block }}$ was varied from $6.0 \times 10^{10}$ to $3.0 \times 10^{10} \mathrm{~cm}^{-2}$. As $\rho_{\text {core-block }}$ decreases from $6.0 \times 10^{10}$ (homogeneous contrast) to $4.0 \times 10^{10} \mathrm{~cm}^{-2}$ (corona contrast), the amplitude of the form factor at low $q$ also decreases due to the reduced contribution from the core. When $\beta_{\text {corel }}\left[=v_{\text {core-block }}\left(\rho_{\text {core-block }}-\rho_{\text {solvent }}\right)\right]$ becomes negative $\left(\rho_{\text {core-block }}=3.5 \times 10^{10}\right.$ and $3.0 \times 10^{10} \mathrm{~cm}^{-2}$, respectively), the amplitudes at low $q$ decrease further, reflecting the negative contribution of the cross term between the core
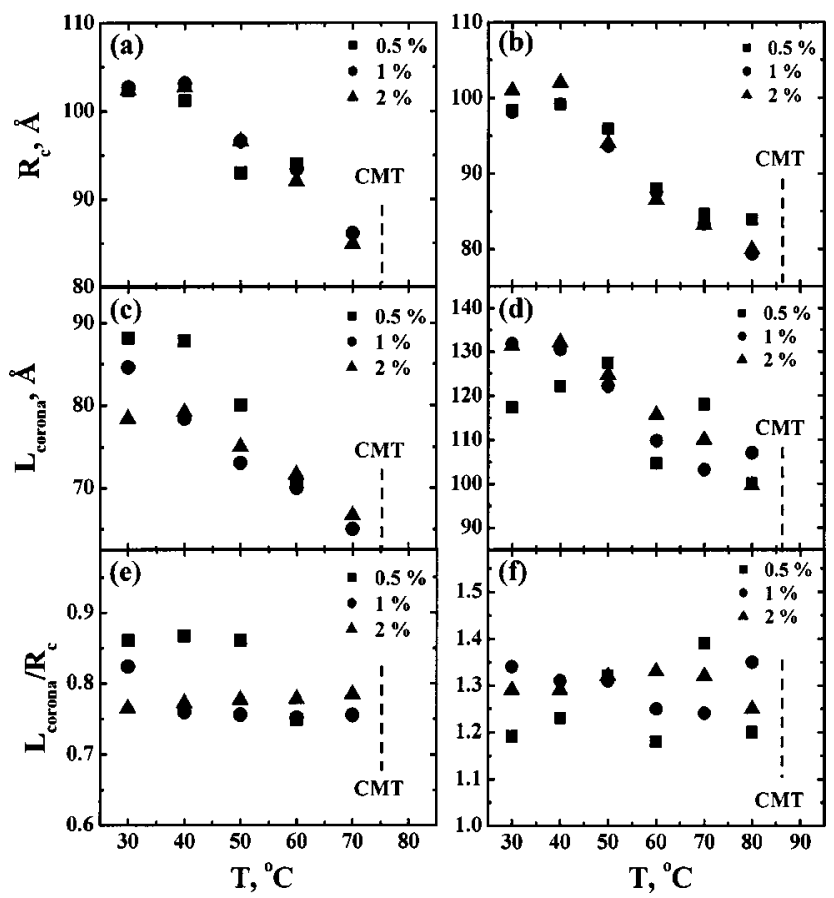

FIG. 5. Temperature dependence of the core radius $R_{c}$ for (a) $\operatorname{SdI}(15-14)$ in DEP and (b) dSI(16-15) in C14, the corona layer thickness $L_{\text {corona }}$, for (c) SdI(15-14) in DEP and (d) dSI(16-15) in C14, and the ratio of the corona layer thickness to the core radius, $L_{\text {corona }} / R_{c}$, for (e) SdI(15-14) in DEP and (f) $\operatorname{dSI}(16-15)$ in C14.

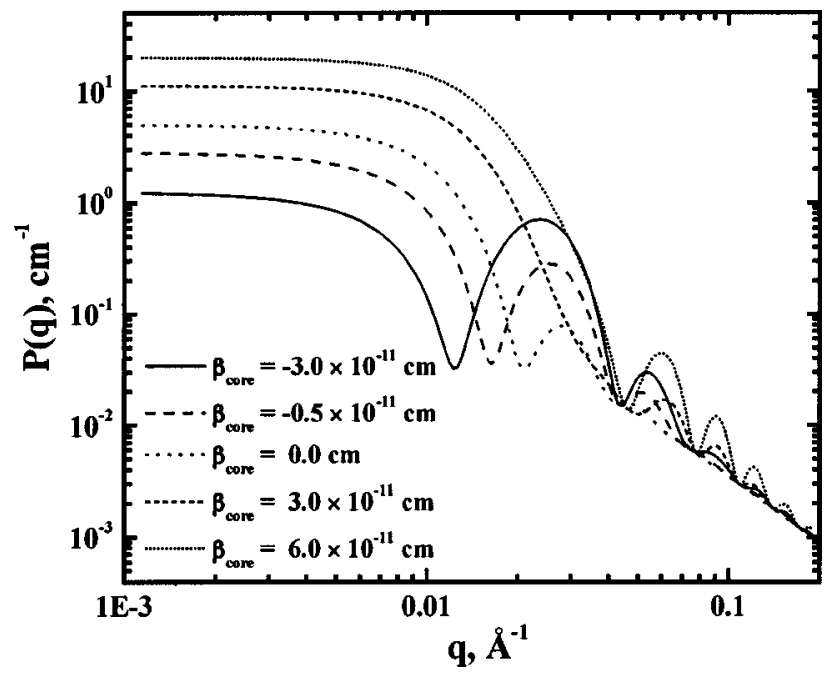

FIG. 6. Calculated form factor for a spherical micelle with $R_{c}=110 \AA, Q$ $=186, R_{g}=45 \AA$, and $d=1$, as a function of core contrast $\beta_{\text {core }}$.

and corona. At the same time, the first maximum of the form factor is clearly magnified and the first minimum appears at lower $q$, consistent with the features in $\operatorname{dSI}(16-15)$ in DEP solutions. In other words, the location of the minimum is very sensitive to the contrast. These features are also seen in other systems. For example, Won et al. investigated poly(oxyethylene- $b$-butadiene) (PEO-PB) diblock copolymers in a mixture of water $\left(\mathrm{H}_{2} \mathrm{O}\right)$ and heavy water $\left(\mathrm{D}_{2} \mathrm{O}\right)$ by SANS. ${ }^{21}$ The contrast was varied by changing the $\mathrm{D}_{2} \mathrm{O}$ content. Between the volume fractions of $\mathrm{D}_{2} \mathrm{O}, \phi_{\mathrm{D}_{2} \mathrm{O}}$, of 0.17 and 0.72 , the cross term between core and corona becomes negative. As the $\mathrm{D}_{2} \mathrm{O}$ content increases $\left(\phi_{\mathrm{D}_{2} \mathrm{O}}=0.48\right.$ and $0.60)$, one can see that the first bump becomes more pronounced (Fig. 7 in Ref. 21). Similar features are also seen in Ref. 15 (Figs. 2, 6, and 7), where $d$-PS-PI diblock copolymers in decane were investigated. ${ }^{15}$

Figures 3(b) and 3(c) display the scattering data and the model fits for SdI(15-14) in C14 and dSI(16-15) in DEP, respectively, as a function of temperature. To describe the corona chains, the data were fitted with a sum of two cubic $b$ spline functions for $\rho_{\text {corona }}(r)$. The model gave an excellent fit to both systems. Especially for dSI(16-15) in DEP, the first sharp minima were nicely captured and the diminution of the secondary bump was also described. Comparing to the core contrast systems, the fitting parameters increase by four, as mentioned previously. The resulting fitting parameters are collected in Table II. For dSI(16-15) in DEP, $Q$ is less than expected compared to the other three systems. For example, with $Q=119$ and $R_{c}=113 \AA$ at $30^{\circ} \mathrm{C}$, the solvent fraction in the core is estimated as $\phi_{\text {core,sol }}=0.45$, while the other three systems predict no solvent in the core at $30^{\circ} \mathrm{C}$. Also, $Q$ shows a significant concentration dependence. We believe this apparent inconsistency is due to the insufficient information in the forward scattering data at low $q$, where $Q$ is directly determined.

As with the core contrast systems, $Q$ and $R_{c}$ decrease at elevated temperature. The micelles in $\mathrm{dSI}(16-15)$ in DEP solutions persist up to higher temperature $\left(\mathrm{CMT} \sim 110^{\circ} \mathrm{C}\right)$, as the molecular weight of $h$-PI in $\mathrm{dSI}(16-15)$ is higher than 

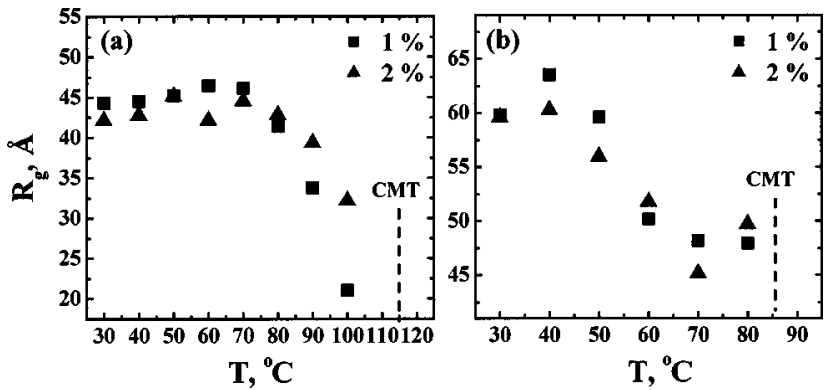

FIG. 7. The radius of gyration of the corona chains, $R_{g}$, as a function of temperature for (a) dSI(16-15) in DEP and (b) SdI(15-14) in C14.

that of $d$-PI in $\operatorname{SdI}(15-14)$. More interestingly, the micellar dimension increases slightly in the range $30-70^{\circ} \mathrm{C}$. At $70{ }^{\circ} \mathrm{C}, R_{c}$ increases by $5.5 \AA(5 \%)$ and $6.8 \AA(6 \%)$ comparing to those of $\phi=0.01$ and 0.02 solutions at $30^{\circ} \mathrm{C}$, respectively, with almost constant $Q$ (equivalently constant intensity at low $q$ in this case), indicating that the micelles are swollen by the solvent. Although the effect is small, it is also consistently observed by DLS [Fig. 9(b)]. While $Q$ is expected to increase with increasing solvent selectivity, the core chains also need to be stretched further with increasing $Q$, which becomes unfavorable due to the entropy penalty of the core chains. Therefore $Q$ may remain constant before it decreases with decreasing solvent selectivity. This regime has been also consistently observed in the concentrated solutions, $\phi=0.2-0.3$, where the micelles pack onto an fcc lattice. $^{28,35}$

The radius of gyration $R_{g}$ of the corona chains is $42-45$ $\AA$ for $d$-PS [dSI(16-15) in DEP], and 59-63 $\AA$ for $d$-PI [SdI (15-14) in C14] at $30^{\circ} \mathrm{C}$. Here we can introduce a dimensionless parameter $s_{\text {corona }}\left(\equiv R_{g} /\left\langle R_{g}\right\rangle_{0 \text {,corona }}\right)$, taken as the ratio of $R_{g}$ of the corona chains to the unperturbed $R_{g}$ of the corona blocks, representing the corona chain stretching. $\left\langle R_{g}\right\rangle_{0 \text {,corona }}$ for $d$-PS and $d$-PI with molecular weights of 15800 and $14100 \mathrm{~g} / \mathrm{mol}$ is 24 and $42 \AA$, respectively. Based on these values, $s_{\text {corona }}$ is $1.6-1.7$ for $d$-PS [dSI(16-15) in DEP] and 1.3-1.4 for $d$-PI [SdI(15-14) in C14] at $30^{\circ} \mathrm{C}$. As the temperature approaches the CMT, the interfacial tension decreases and both core and corona chains are allowed to relax. $R_{g}$ near the CMT becomes close to $\left\langle R_{g}\right\rangle_{0 \text {,corona }}$, i.e., 21-32 $\AA$ for $d$-PS and 48-50 $\AA$ for $d$-PI, respectively, as shown in Fig. 7. Castelletto et al. also observed a decrease in $R_{g}$ of the corona chains with increasing temperature in poly(oxyethylene- $b$-oxybutylene) (PEO-PBO) diblock copolymer in $\mathrm{D}_{2} \mathrm{O} .{ }^{13}$ Seemingly, it may look like the same phenomenon, but the origin is in fact quite different. In their work, the corona PEO chains actually contract as the solvent quality becomes worse with increasing temperature, whereas the corona chains are always under a good solvent condition in our systems.

The density profile of the corona chains, $\rho_{\text {corona }}(r)$, can be determined from the fitting parameters $R_{c}, s$, and $a_{1}$. $\rho_{\text {corona }}(r)$ was rescaled as follows:

$$
\int 4 \pi \hat{\rho}_{\text {corona }}(r) r^{2} d r=Q v_{\text {corona-block }},
$$
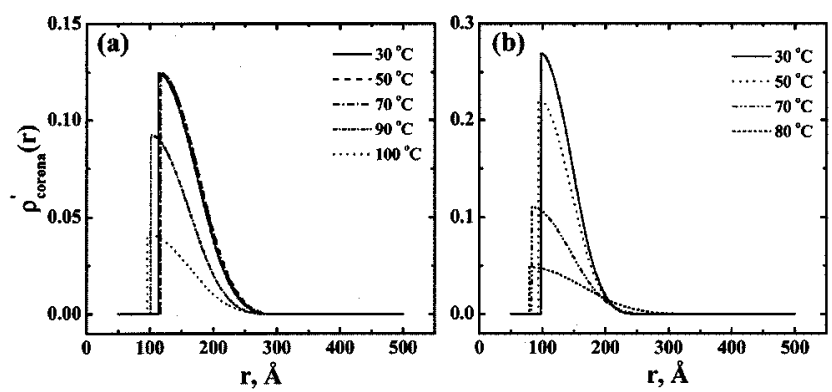

FIG. 8. The radial profile for the corona as a function of temperature for (a) $\mathrm{dSI}(16-15)$ in DEP $\phi=0.01$ and (b) SdI(15-14) in C14 $\phi=0.01$.

where $\hat{\rho}_{\text {corona }}(r)$ is the rescaled corona chain profile and represents the volume fraction of the corona chains. Figure 8 displays the corresponding corona chain profiles for $\phi=0.01$ solutions of dSI(16-15) in DEP and $\operatorname{SdI}(15-14)$ in C14 at selected temperatures. The maximum volume fraction of the corona chain is below 0.3 , consistent with other results in the literature. ${ }^{13-17}$ Note that $\hat{\rho}_{\text {corona }}(r)$ of $\operatorname{dSI}(16-15)$ in DEP is less than that of SdI(15-14) in C14 due to the underestimated $Q$. As temperature increases, the width of the corona profile decreases and the maximum of the volume fraction decreases. A decrease in the maximum of the volume fraction reflects the dilution of the corona, due to the decrease in $Q$ as well as the core swelling by solvent.

\section{Comparison to DLS ( $\boldsymbol{R}_{h s}$ versus $\boldsymbol{R}_{h}$ )}

The hydrodynamic radii of the micelles and single chains across the CMT were measured by DLS for each system, and $R_{h}$ of the micelles was compared to $R_{h s}$ from model fitting of the SANS data (Fig. 9). The CMT from DLS can be measured by a combination of an abrupt change in $R_{h}$ and the scattered intensity, and that from SANS can be predicted by a significant decrease in the low $q$ intensity of single chains (data not shown). As indicated in Fig. 9, the CMTs from both measurements match very well. Also, the agree-
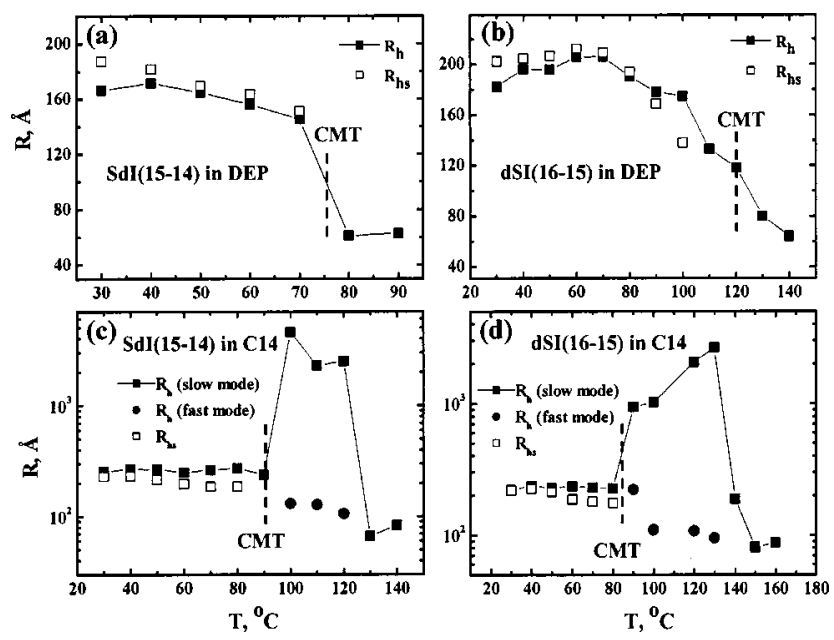

FIG. 9. The hard-sphere radius $R_{h s}$ and the hydrodynamic radius, $R_{h}$, as a function of temperature for (a) SdI(15-14) in DEP $\phi=0.01$, (b) $\mathrm{dSI}(16-15)$ in DEP $\phi=0.01$, (c) dSI(16-15) in C14 $\phi=0.01$, and (d) SdI(15-14) in C14 $\phi=0.01$. 
ment between $R_{h}$ from DLS and $R_{h s}$ from SANS is excellent, considering the very different origins of these quantities; $R_{h}$ is calculated through the Stokes-Einstein equation using the diffusion coefficient measured from DLS and the solvent viscosity, and $R_{h s}$ is determined from the SANS structure factor.

Another noticeable feature is the "anomalous micellization," which emerges above the real CMT in C14 solutions. We have demonstrated previously that the anomalous micellization is due to the incipient phase separation of small quantities of PS homopolymer, resulting from incomplete crossover to the second block during the sequential living anionic polymerization. ${ }^{36}$ The intensity correlation functions in this regime can be fitted to a sum of two exponentials; the faster mode corresponds to the single chains and the slower model is due to the large assemblies, which we proposed to be emulsionlike droplets. In Figs. 9(c) and 9(d), the anomalous micellization regime appears as two dynamic modes above the real CMT. The real CMT was confirmed again by SANS in the sense that no evidence of micelles was observed in the SANS data above the real CMT. Moreover, there is no signature of large structures, because the associated $R_{h}$ is typically greater than $1000 \AA$ and would therefore appear well below the low $q$ limit in the SANS measurements.

\section{DISCUSSION}

Previous studies have focused on aqueous systems (i.e., PEO-based copolymers in water), ${ }^{4-13,15-17}$ and, to the best of our knowledge, this is the first report of a systematic SANS study of the temperature-dependent micellar structures in organic solvents. Therefore, it should be worthwhile to compare our results with those in aqueous systems, in view of the changes in solvent selectivity accessible in organic solvents. The polymers most frequently used in aqueous systems were poly(oxyethylene- $b$-oxypropylene- $b$-oxyethylene) (PEOPPO-PEO) triblock copolymers, denoted $\mathrm{PEO}_{m} \mathrm{PPO}_{n} \mathrm{PEO}_{m}$, where $m$ and $n$ represent degrees of polymerization. As temperature decreases, the solvent quality for the PEO blocks increases, and the hydrophobicity in both core and corona blocks decreases, resulting in a decrease in the interfacial tension. In this case, the CMT is found upon cooling, i.e., a lower critical solution temperature (LCST) system. Regardless of the fitting models adopted, all the results in the literature showed consistently that $Q$ decreases and $\phi_{\text {sol,core }}$ increases with decreasing temperature, as described below. This is in good agreement with our results in that $Q$ decreases and $\phi_{\text {sol,core }}$ increases with decreasing interfacial tension, as the CMT is approached.

Early work on the micellar behavior in aqueous systems was performed by Mortensen and Pedersen. ${ }^{4}$ $\mathrm{PEO}_{25} \mathrm{PPO}_{40} \mathrm{PEO}_{25}$ in $\mathrm{D}_{2} \mathrm{O}$ at various concentrations was characterized by SANS, using a model that assumed the micelle was a homogeneous core with no solvent, and the corona was not included. From the model fits they found that the micellar size (comparable to $R_{c}$ ) and $Q$ increased with increasing temperature. Mortensen and Brown investigated a series of PEO-PPO-PEO triblock copolymers in $\mathrm{D}_{2} \mathrm{O}$ using the same model. ${ }^{5}$ The block copolymers were selected to have a common degree of polymerization of the inner PPO blocks, but different degrees of polymerization of the outer PEO blocks. The main result was that $R_{c}$ increases with decreasing degree of polymerization of the PEO blocks, and with increasing temperature. Also, the CMT was found to increase with increasing PPO concentration.

Goldmints et al. studied the micellar structures near the CMT for $\mathrm{PEO}_{26} \mathrm{PPO}_{40} \mathrm{PEO}_{26}$ and $d-\mathrm{PEO}_{23} \mathrm{PPO}_{34} d-\mathrm{PEO}_{23}$ in mixtures of $\mathrm{H}_{2} \mathrm{O}$ and $\mathrm{D}_{2} \mathrm{O} .{ }^{6,7}$ A model consisting of a homogeneous core and corona was used to fit the SANS data. This model allows the presence of solvent in both the micelle core and the corona, whereas a spatial variation in the corona concentration cannot be described. The micellar structures were compared in terms of $T-\mathrm{CMT}$, as the CMT also depended on concentration in these systems. Over the temperature range $T-\mathrm{CMT} \leqslant 10^{\circ} \mathrm{C}$, it was shown that the water content in the core decreases and $Q$ increases with increasing temperature, while $R_{c}$ is nearly constant. Also, all values were essentially independent of the concentration, and the authors suggested that it is the distance from the CMT that determines the micellar structure in this region.

With the same model, Alexandridis and Yang described $\mathrm{PEO}_{37} \mathrm{PPO}_{58} \mathrm{PEO}_{37}$ in various mixtures of water and nonaqueous polar solvents: formamide, ${ }^{8}$ water/formamide,, 10 water/ethanol, ${ }^{10}$ and water/glycerol. ${ }^{10}$ They measured the CMT as a function of concentration and water/solvent ratio, and characterized the temperature-dependent micellar structures. Although the details differed depending on the solvent quality of the nonaqueous solvent, the overall features are very similar to those in water in the context of temperaturedependent micellar behavior.

In the work of Liu et al., a "cap and gown" model was proposed to incorporate a radial distribution of corona chains. ${ }^{12}$ This model considers a homogeneous core and the diffuse corona layer, and satisfactorily described the micelles of PEO-PPO-PEO triblock copolymers in $\mathrm{D}_{2} \mathrm{O}$ at various concentrations and temperatures. The micellar structure remained constant as a function of concentration, but varied with temperature, consistent with other results.

More recently, Castelletto et al. used the form factor for the block copolymer micelles developed by Pedersen and co-workers to investigate PEO-PBO diblock copolymers in $\mathrm{D}_{2} \mathrm{O}{ }^{13,17}$ The polymers were chosen to have long corona chains, and the radial profiles of the corona chains $\rho_{\text {corona }}(r)$ were described by a sum of two cubic $b$ spline functions, as also used in this paper. The benefit of this expression compared to others is that the Fourier transformation gives rise to a fully analytical expression, and hence the number of numerical integrations can be minimized in the fitting procedure. As with previous models, the fits indicated that $Q$ increases and $\phi_{\text {core,sol }}$ decreases with increasing temperature. Also, the maximum volume fraction in $\rho_{\text {corona }}(r)$ decreases with decreasing temperature, analogous to our systems in reflecting the dilution of the corona due to the decrease in $Q$ and the core swelling by solvent. In addition, $R_{g}$ in the corona decreases with increasing temperature as with our systems, but due to different reason as described above.

No significant concentration dependence of the micellar dimensions was reported, except relatively small deviations. 
Based on this, Castelletto et al. adopted the same approach as we did subsequently, in relating the micellar dimensions in the dilute solutions to those in the ordered states for PEOPBO diblock copolymer solutions. ${ }^{17}$ In their work, $R_{h s}$ in dilute solution showed good agreement with the nearestneighbor distance in a bcc lattice, as we also found. ${ }^{28}$ The success of this approach implies that the micellar characteristics in dilute solutions also reflect those in the higher concentration solutions, thus providing a crucial key to interpreting the phase diagram. For example, we showed that in the dilute solutions $L_{\text {corona }} / R_{c}$ remains unchanged across the relevant temperature range, whereas $Q$ decreases with increasing temperature. This fact supports the interpretation that the fcc to bcc transition in concentrated solutions is induced by a decrease in $Q$, not by a certain value of $L_{\text {corona }} / R_{c}$. Another example is a "reentrant" ordering transition from the disordered micelles to the fcc or bcc lattices upon heating, reported in some solutions. ${ }^{33,34,42}$ We postulated that this is due to an increase in the micellar size due to the core swelling. In dSI(16-15) in DEP solutions, we have shown that $R_{c}$ and $R_{h s}$ increase slightly with constant $Q$ over the temperature range $30-70{ }^{\circ} \mathrm{C}$, and thus this provides quantitative support for the previous hypothesis.

\section{SUMMARY}

To understand how changing solvent selectivity controls micellar structure, we characterized the detailed micellar characteristics with varying temperature by using SANS. $\mathrm{SdI}(15-14)$ in DEP, dSI(16-15) in C14, dSI(16-15) in DEP, and $\mathrm{SdI}(15-14)$ in $\mathrm{C} 14$ represent the core contrast systems with $d$-PI and $d$-PS cores, and the corona contrast systems with $d$-PS and $d$-PI coronas, respectively. The SANS data were obtained at low concentrations so that the effect of intermicellar correlations was minimized, even though the structure factor was incorporated in the model fit.

As temperature increases, the solvent selectivity decreases and the solvent penetrates the core. This is accompanied by a decrease in $Q$ and $R_{c}$, resulting in more diffuse micelles. The corona chains also relax and approach the unperturbed $R_{g}$ of the corona chains near the CMT, as the interfacial tension decreases with increasing temperature. There is a low temperature regime where $Q$ is constant. This regime persists up to higher temperature $\left(\sim 70^{\circ} \mathrm{C}\right)$ for $\mathrm{dSI}(16-15)$ in DEP which has the highest CMT $\left(\sim 110^{\circ} \mathrm{C}\right)$. In this case, $R_{c}$ increases by $5 \%-6 \%$ with increasing temperature, indicating a regime of core swelling by the solvent at constant $Q$. These results provide the quantitative information necessary to understand the thermoreversible fcc to bcc transition observed at higher concentrations in the same systems.

\section{ACKNOWLEDGMENTS}

This work was supported primarily by the National Science Foundation, through the University of Minnesota MRSEC (Grant No. DMR-0212302), and also in part by the Brain Korea 21 Program endorsed by the Korean Ministry of Education. Further support for this research was provided by the National Institute of Standards and Technology, U.S. De-

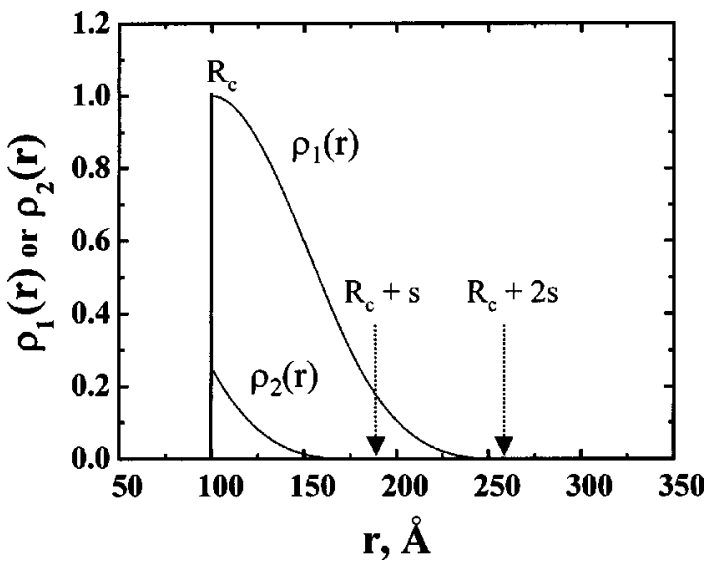

FIG. 10. The radial profiles for two cubic $b$ splines, $\rho_{1}(r)$ and $\rho_{2}(r)$, in $\rho_{\text {corona }}(r)$ with $R_{g}=100 \AA$ and $s=80 \AA$.

partment of Commerce, through the neutron research facilities. We also thank Dr. Jan Skov Pedersen for helpful discussions.

\section{APPENDIX: RADIAL PROFILE OF THE CORONA}

In this work the radial profile of the corona chains, $\rho_{\text {corona }}(r)$, was described as a linear combination of two expressions, $\rho_{1}(r)$ and $\rho_{2}(r) .^{15}$ The profile is then

$$
\rho_{\text {corona }}(r)=\frac{\rho_{1}(r)+a_{1} \rho_{2}(r)}{1+a_{1}},
$$

where $a_{1}$ is a fitting parameter. $\rho_{1}(r)$ and $\rho_{2}(r)$ are

$$
\begin{aligned}
& \rho_{1}(r)= \frac{4\left(r-R_{c}-s\right)^{3}-\left(r-R_{c}-2 s\right)^{3}}{4 s^{3}} \\
& \text { for } R_{c} \leqslant r<R_{c}+s, \\
& \rho_{1}(r)= \frac{-\left(r-R_{c}-2 s\right)^{3}}{4 s^{3}} \text { for } R_{c}+s \leqslant r<R_{c}+2 s, \\
& \rho_{1}(r)=0 \quad \text { elsewhere }
\end{aligned}
$$

and

$$
\begin{aligned}
& \rho_{2}(r)=\frac{-\left(r-R_{c}-s\right)^{3}}{4 s^{3}} \text { for } R_{c} \leqslant r<R_{c}+s, \\
& \rho_{2}(r)=0 \quad \text { elsewhere. }
\end{aligned}
$$

The parameter $s$ gives the width of the profile. In our case, $s$ is about twice the radius of gyration of the corona chains. Figure 10 shows an example of $\rho_{1}(r)$ and $\rho_{2}(r)$ with $R_{c}$ $=100 \AA$ and $s=80 \AA$. While $s$ changes the width of the corona profiles, $a_{1}$ adjusts the amplitude of the profile by a linear combination of $\rho_{1}(r)$ and $\rho_{2}(r)$. The Fourier transform of $\rho_{1}(r)$ and $\rho_{2}(r)$ can be calculated separately as follows: 


$$
\begin{aligned}
S_{1}(q)= & C_{\text {norm }, 1}\left[\frac{24 \cos \left[q\left(R_{c}+2 s\right)\right]}{q^{6}}\right. \\
& +\frac{6\left(R_{c}+2 s\right) \sin \left[q\left(R_{c}+2 s\right)\right]}{q^{5}} \\
& -\frac{96 \cos \left[q\left(R_{c}+s\right)\right]}{q^{6}}-\frac{24\left(R_{c}+s\right) \sin \left[q\left(R_{c}+s\right)\right]}{q^{5}} \\
& +\frac{4\left(q^{4} R_{c} s^{3}+3 q^{2} R_{c} s+18\right) \cos \left(q R_{c}\right)}{q^{6}} \\
& \left.-\frac{\left.2\left[2 q^{2} s^{3}-9\left(R_{c}-2 s\right)\right] \sin \left(q R_{c}\right)\right]}{q^{5}}\right]
\end{aligned}
$$

where

$$
C_{\text {norm }, 1}^{-1}=\frac{s^{4}\left(15 R_{c}^{2}+14 R_{c} s+5 s^{2}\right)}{5}
$$

and

$$
\begin{aligned}
S_{2}(q)= & C_{\text {norm }, 2}\left[\frac{96 \cos \left[q\left(R_{c}+2 s\right)\right]}{q^{6}}\right. \\
& +\frac{24\left(R_{c}+2 s\right) \sin \left[q\left(R_{c}+2 s\right)\right]}{q^{5}} \\
& +\frac{4\left(q^{4} R_{c} s^{3}-6 q^{2} s\left(R_{c}-s\right)-24\right) \cos \left(q R_{c}\right)}{q^{6}} \\
& \left.+\frac{4\left[q^{2} s^{2}\left(3 R_{c}-s\right)-6\left(R_{c}-3 s\right)\right] \sin \left(q R_{c}\right)}{q^{5}}\right],
\end{aligned}
$$

where

$$
C_{\mathrm{norm}, 2}^{-1}=\frac{s^{4}\left(15 R_{c}^{2}+6 R_{c} s+s^{2}\right)}{15} .
$$

The corona scattering amplitude $A_{\text {corona }}(q)$ can be written as

$$
A_{\text {corona }}(q)=\frac{S_{1}(q)+a_{1} S_{2}(q)}{1+a_{1}} \exp \left(-q^{2} \sigma^{2} / 2\right) \text {. }
$$

${ }^{1}$ I. W. Hamley, The Physics of Block Copolymers (Oxford University Press, New York, 1998).

${ }^{2}$ Z. Tuzar and P. Kratochvil, Surf. Colloid Sci. 15, 1 (1993).

${ }^{3}$ A. Halperin, M. Tirrell, and T. P. Lodge, Adv. Polym. Sci. 100, 33 (1992).

${ }^{4}$ K. Mortensen and J. S. Pedersen, Macromolecules 26, 805 (1993).

${ }^{5}$ K. Mortensen and W. Brown, Macromolecules 26, 4128 (1993).

${ }^{6}$ I. Goldmints, F. K. Von Gottberg, K. A. Smith, and T. A. Hatton, Langmuir 13, 3659 (1997).
${ }^{7}$ I. Goldmints, G.-E. Yu, C. Booth, K. A. Smith, and T. A. Hatton, Langmuir 15, 1651 (1999).

${ }^{8}$ P. Alexandridis and L. Yang, Macromolecules 33, 3382 (2000).

${ }^{9}$ L. Yang and P. Alexandridis, Langmuir 16, 4819 (2000).

${ }^{10} \mathrm{P}$. Alexandridis and L. Yang, Macromolecules 33, 5574 (2000).

${ }^{11}$ L. Lobry, N. Micali, F. Mallamace, C. Liao, and S. H. Chen, Phys. Rev. E 60, 7076 (1999).

${ }^{12}$ Y. Liu, S.-H. Chen, and J. S. Huang, Macromolecules 31, 2236 (1998).

${ }^{13}$ V. Castelletto, I. W. Hamley, and J. S. Pedersen, J. Chem. Phys. 117, 8124 (2002).

${ }^{14}$ J. S. Pedersen and M. C. Gerstenberg, Colloids Surf., A 213, 175 (2003).

${ }^{15}$ J. S. Pedersen, C. Svaneborg, K. Almdal, I. W. Hamley, and R. N. Young, Macromolecules 36, 416 (2003).

${ }^{16}$ C. Sommer and J. S. Pedersen, Macromolecules 37, 1682 (2004).

${ }^{17}$ V. Castelletto, I. W. Hamley, and J. S. Pedersen, Langmuir 20, 2992 (2004).

${ }^{18}$ J. S. Pedersen and C. Svaneborg, Curr. Opin. Colloid Interface Sci. 7, 158 (2002).

${ }^{19}$ S. Foerster, E. Wenz, and P. Lindner, Phys. Rev. Lett. 77, 95 (1996).

${ }^{20}$ L. Willner, A. Poppe, J. Allgaier, M. Monkenbusch, P. Lindner, and D. Richter, Europhys. Lett. 51, 628 (2000).

${ }^{21}$ Y.-Y. Won, H. T. Davis, F. S. Bates, M. Agamalian, and G. D. Wignall, J. Phys. Chem. B 104, 7134 (2000).

${ }^{22}$ J. S. Pedersen and M. C. Gerstenberg, Macromolecules 29, 1363 (1996).

${ }^{23}$ J. S. Pedersen, J. Appl. Crystallogr. 33, 637 (2000).

${ }^{24}$ J. S. Pedersen, I. W. Hamley, C. Y. Ryu, and T. P. Lodge, Macromolecules 33, 542 (2000).

${ }^{25}$ J. S. Pedersen, J. Chem. Phys. 114, 2839 (2001).

${ }^{26}$ C. Svaneborg and J. S. Pedersen, J. Chem. Phys. 112, 9661 (2000).

${ }^{27}$ C. Svaneborg and J. S. Pedersen, Macromolecules 35, 1028 (2002).

${ }^{28}$ T. P. Lodge, J. Bang, M. J. Park, and K. Char, Phys. Rev. Lett. 92, 145501 (2004).

${ }^{29}$ G. A. McConnell, A. P. Gast, J. S. Huang, and S. D. Smith, Phys. Rev. Lett. 71, 2102 (1993).

${ }^{30}$ G. A. McConnell, M. Y. Lin, and A. P. Gast, Macromolecules 28, 6754 (1995).

${ }^{31}$ M. Watzlawek, C. N. Likos, and H. Löwen, Phys. Rev. Lett. 82, 5289 (1999).

${ }^{32}$ B. Groh and M. Schmidt, J. Chem. Phys. 114, 5450 (2001).

${ }^{33}$ T. P. Lodge, B. Pudil, and K. J. Hanley, Macromolecules 33, 5918 (2002).

${ }^{34}$ J. Bang, T. P. Lodge, X. Wang, K. L. Brinker, and W. R. Burghardt, Phys. Rev. Lett. 89, 215505/1 (2002).

${ }^{35}$ J. Bang and T. P. Lodge, J. Phys. Chem. B 107, 12071 (2003).

${ }^{36}$ T. P. Lodge, J. Bang, K. J. Hanley, J. Krocak, S. Dahlquist, B. Sujan, and J. Ott, Langmuir 19, 2103 (2003).

${ }^{37}$ N. W. Ashcroft and J. Lekner, Phys. Rev. 145, 83 (1966).

${ }^{38}$ J. S. Pedersen, J. Appl. Crystallogr. 27, 595 (1994).

${ }^{39}$ M. Antonini, A. Daneri, and G. Toselli, J. Appl. Crystallogr. 3, 145 (1970).

${ }^{40}$ J. S. Pedersen, D. Posselt, and K. Mortensen, J. Appl. Crystallogr. 23, 321 (1990).

${ }^{41}$ J. G. Barker and J. S. Pedersen, J. Appl. Crystallogr. 28, 105 (1995).

${ }^{42}$ K. J. Hanley, T. P. Lodge, and C.-I. Huang, Macromolecules 33, 5918 (2000).

${ }^{43}$ I. W. Hamley, J. P. A. Fairclough, A. J. Ryan, C. Y. Ryu, T. P. Lodge, A. J. Gleeson, and J. S. Pedersen, Macromolecules 31, 1188 (1998).

${ }^{44}$ K. J. Hanley and T. P. Lodge, J. Polym. Sci., Polym. Phys. Ed. 36, 3101 (1998).

${ }^{45}$ M. J. Park, J. Bang, T. Harada, T. P. Lodge, and K. Char, Macromolecules (to be published).

${ }^{46}$ I. W. Hamley, J. A. Pople, and O. Diat, Colloid Polym. Sci. 276, 446 (1998). 